\title{
EL CEREBRO HUMANO, UNA PERSPECTIVA TRANSDISCIPLINARIA
}

\author{
THE HUMAN BRAIN, A TRANSDISCIPLINARY PERSPECTIVE
}

\author{
${ }^{1}$ Carlos Cuya Mamani
}

\begin{abstract}
RESUMEN
El articulo hace una revisión sobre el estudio del cerebro en los diversos campos de la actividad cientifica. Pone énfasis en que dicho conocimiento no es ya patrimonio de las ciencias de la salud, sino que tiene un alcance cultural más amplio. Analiza el cerebro desde el punto de vista de las actividades mentales del ser humano, asi como desde el punto de vista social.
\end{abstract}

Palabras Clave: Cerebro humano, perspectivas.

ABSTRACT

The paper offers an overview on the study of the brain in various fields of scientific activity. It emphasizes that such knowledge is no longer patrimony of health sciences, but has a broader cultural scope. It analyzes the brain from the point of view of human mental activities as well as from the social point of view.

Key Words: Brain, prospects.

\section{INTRODUCCIÓN}

Para construir el cerebro humano, la evolución de las especies ha necesitado casi mil quinientos millones de años. La aparición de las neuronas o células cerebrales fue uno de los acontecimientos más importantes de la evolución de los seres pluricelulares. Así, el cerebro humano emergió hace unos doscientos mil años junto con nuestra especie, luego de una larga travesía, desde entonces no ha evolucionado estructuralmente. Y resulta realmente paradójico que durante tanto tiempo este órgano, del que somos inseparables y gracias al cual somos los que somos, haya sido un desconocido para nosotros. Su descubrimiento y exploración son posteriores a los de América. Antes, esta terra incognita era sólo materia de especulación y superstición.

Es comprensible, en la actualidad, que de todos los objetos naturales y artificiales que existen en el universo, el cerebro del hombre es de lejos el más complejo. Resulta pues, inadecuada la analogía entre el cerebro y el ordenador, éste sigue $\sin$ tener un equivalente en informática. La complejidad cerebral se expresa, por un lado, por la yuxtaposición de territorios muy diferentes cuyas funciones están más o menos bien especificadas y, por otro, por la cantidad de células nerviosas: cien mil millones de neuronas y muchas más células gliales. Aumentan esta complejidad una cantidad significativa de contactos o sinapsis entre células nerviosas. Una neurona recibe (o es el origen) de aproximadamente diez mil contactos, lo que hace miles de billones de conexiones entre neuronas, mediante los cuales intercambia, de forma permanente, señales eléctricas y químicas con otras células para captar, tratar y almacenar informaciones pertinentes que permitirán la adaptación del sujeto a su entorno.

Los estudios anatómicos y fisiológicos nos muestran que el cerebro, que pesa 1,400 gramos, comprende dos regiones diferenciadas. La parte más externa del cerebro, la corteza cerebral, es poco sensible a la dictadura de los genes, se ve constantemente remodelada por la experiencia de la persona. Sus redes neuronales son muy influenciadas por factores del ambiente y del mismo individuo las cuales permiten regulaciones denominadas epigenéticas. Por el contrario, las regiones más profundas del cerebro - el cerebro basal - reaccionan anatómicamente mucho menos a los estímulos del medio ambiente y a las experiencias del sujeto. Estas zonas permanecen estables, genéticamente especificadas y poseen un origen evolutivo antiguo.

A nivel individual, el cerebro permite las actividades mentales del ser humano, y han sido tratadas brillantemente por Alexis Carrel, médico y Premio Nóbel de Medicina en 1912, en su libro La incógnita del hombre'. Estas comprenden: las actividades intelectuales o cognitivas, las actividades éticas o morales, el sentido estético y las actividades místicas. Pero es necesario aclarar, que las actividades mentales dependen de las actividades fisiológicas, es decir, de las influencias que ejercen el resto de sistemas del organismo, como por ejemplo las hormonas que producen los diversos órganos endocrinos.

El cerebro es también un órgano social, pues lo que nos hace ser humanos no es sólo esa deslumbrante capacidad cognitiva ni nuestro enorme cerebro, sino 
también esa virtud para participar de interacciones sociales.

Aunque esta división en el estudio del cerebro es artificial, se hace necesaria para poder establecer y precisar estas características tan admirables. Es preciso señalar que el conocimiento desarrollado acerca del cerebro ya no es patrimonio de las ciencias de la salud, en especial de la neurología o neurocirugía, lo es de toda la cultura humana, pues su aplicación compete a todos los campos del desarrollo humano, hablándose en la actualidad de neurocultura.

\section{DESARROLLO TEMÁTICO}

\subsection{La Neurociencia y el estudio del cerebro}

"Dentro de los 4000 millones de años de historia de la vida sobre nuestro planeta, dentro de los 4 millones de años de bistoria de la familia bumana, hay una sola generación privilegiada que podrá vivir este momento único de transición: la muestra", escribía Carl Sagan en su libro El cerebro de Broca?. Y es verdad, pues somos testigos de deslumbrantes descubrimientos que nos permiten, en cierta forma, empezar a responder una de las eternas preguntas filosóficas: ¿Quiénes somos? El descubrimiento de la estructura del $\mathrm{ADN}$, los aportes de la biología celular y molecular, nos ha permitido acceder a un mundo desconocido que la evolución y la naturaleza habían hecho casi inaccesibles, pero que la curiosidad humana permitió desentrañar. Por primera vez en la historia de la humanidad, el hombre empieza a conocerse.

El desafío central de la ciencia en el siglo XXI es la comprensión de la mente humana en términos biológicos. La posibilidad de salir triunfantes de ese desafio surgió a finales del siglo XX, al unificarse la psicología cognitiva -la ciencia de la mente- y la neurociencia - la ciencia del cerebro- El resultado fue una ciencia de la mente que nos ha permitido contestar un conjunto de preguntas sobre nosotros mismos?.

El vertiginoso desarrollo de las ciencias de la física y las técnicas de neuroimagen, como la tomografía axial computarizada, la tomografía de emisión de positrones, la resonancia magnética nuclear y otras han permitido una explosión en el conocimiento del cerebro ${ }^{4}$. Esto aunado a que en diversos países, como Estados Unidos de Norteamérica, hayan puesto a disponibilidad recursos económicos para este propósito, como ocurrió con la llamada Década del Cerebro, en el último decenio del siglo pasado.

\subsection{Lóbulos frontales: estructuras recientemente evolucionadas del cerebro}

El cerebro humano es el sistema natural más complejo del universo conocido. Es el nuevo reto de la ciencia. Más recientemente, la neurociencia, que estudia el cerebro, está permitiendo conocer la importancia y trascendencia que este órgano tiene en la vida del hombre y del universo. Aunque en general podemos decir que la corteza cerebral, una delgada capa de células nerviosas, permitió al ser humano muchas capacidades, fue finalmente el desarrollo de sus lóbulos frontales, lo que le permitió crear la civilización ${ }^{5}$. Los lóbulos frontales son pues el último logro en la evolución del sistema nervioso: solo en los seres

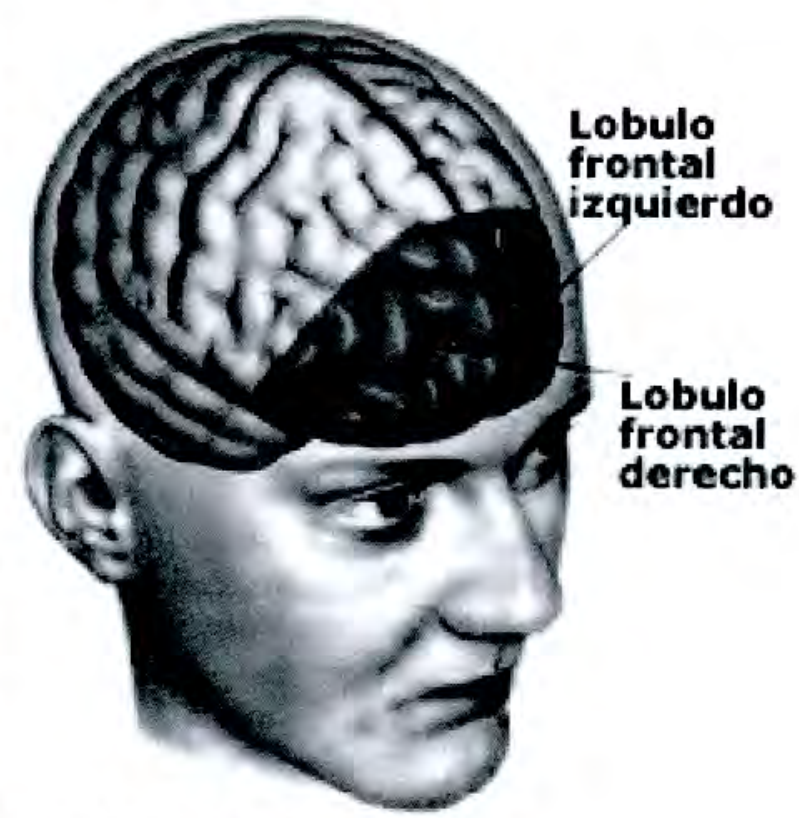

Figura $\mathrm{N}^{\circ} 01$. Lóbulos frontales

humanos ( $y$, en alguna medida, en los grandes simios) alcanzan un desarrollo tan grande.

Los lóbulos frontales son esenciales para cualquier comportamiento de orden superior: identificar objetivos, proyectar metas y establecer planes para alcanzarla, organizar los medios, llevar a efecto dichos planes, controlar y juzgar los resultados para ver que todo se ha llevado a cabo tal como se pretendía. Cuán importantes resultan estas capacidades en la vida del ser humano. La intencionalidad de la persona reside en los lóbulos frontales, y estos son cruciales para la consciencia superior, para el juicio, para la imaginación, para la empatia, para la identidad, en general para el "alma".

Las potencias cognitivas dependen de los lóbulos frontales y evolucionan con ellos. Estos dotan al organismo de la capacidad de crear modelos neurales de cosas, que son prerrequisitos para hacer que las cosas sucedan. Asi, debe adquirir la capacidad de manipular y transformar dichos modelos, debe adquirir la capacidad de trabajar con representaciones mentales.

Asimismo, la capacidad de cambiar con facilidad de una actividad o idea a otra es tan natural y automática que la damos por supuesta. Esto, de hecho, requiere una estructura neurológica compleja, que también depende de los lóbulos frontales. La flexibilidad mental, la capacidad de ver las cosas con una nueva perspectiva, la creatividad y la originalidad dependen también de los lóbulos frontales. Muchas veces vemos personas rígidas mentalmente en las diversas actividades humanas que impiden el normal desempeño de una organización social.

El éxito en la vida depende principalmente de dos capacidades: la capacidad para darnos cuenta de nuestro propio mundo interior o mental y el de otras personas (empatía). Estas capacidades están estrechamente interrelacionadas y ambas están bajo el control del lóbulo frontal. Son importantes en el control emocional y el de los 
impulsos. He ahí la importancia del lóbulo frontal y del cerebro mismo como órgano social.

Cuando los lóbulos frontales enferman producen alteraciones que tienen que ver con lo esencial del ser humano, con su propia personalidad, y estas alteraciones varían con la gravedad del daño. En el síndrome orbitofrontal son emocionalmente desinhibidos, no tienen previsión de la consecuencia de sus acciones, estas muchas veces son antisociales. En el síndrome dorsolateral, existe una incapacidad para iniciar cualquier comportamiento; una vez comprometido en un comportamiento, el paciente es incapaz de concluirlo o cambiarlo para sí mismo.

\subsection{El cerebro individual}

\section{Cerebro intelectual}

Las funciones ejecutivas son procesos cognitivos que organizan pensamientos, ideas y acciones con un fin. Son la base del intelecto, la personalidad, la conciencia, la conducta social y la empatía. Son también una amalgama de habilidades relacionadas con la planificación, la formación de conceptos, el pensamiento abstracto, la toma de decisiones, la flexibilidad cognitiva, el uso de la retroalimentación, la organización temporal de eventos, la inteligencia general o fluida, y el seguimiento de las acciones.

Al lóbulo frontal se le atribuye las capacidades enumeradas arriba, que pueden ser circunscritas como capacidad ejecutiva o directiva de la conducta humana.

\section{Cerebro ético/moral}

Según Patricia Churchland, "la bipótesis más actual es que 10 que nosotros denominamos "ética" o "moralidad" es una estructura de conducta social en cuatro dimensiones determinada por la interrelación de distintos procesos cerebrales: (1) El cuidado a la atención a los demás (enraizado en el apego a muestros familiares y la preocupación por su bienestar), (2) el reconocimiento de los estados psicológicos de los demás (basado en las ventajas de predecir la conducta de terceros), (3) la resolución de problemas en un contexto social (por ejemplo, cómo deberiamos distribuir los bienes cuando son escasos, como resolver disputas territoriales a cómo deberiamos castigar a los bribones) y (4) el aprendizaje de prácticas sociales (mediante un refuerzo positivo y negativo, por imitación, por ensayo y error, por diversos condicionamientos y por analogia). I a sencillez de esta estructura no significa que sus formas, variaciones y mecanismos neuronales sean simples. Al contrario, la vida social es increiblemente compleja, puesto que el cerebro es el órgano que la administra".

Debemos distinguir dos aspectos esenciales de la neuroética; por un lado la ética de la neurociencia y por otro la neurociencia de la ética.

Para Michael Gazzaniga ${ }^{\star}$ la ética de la neurociencia es "...el examen de cómo queremos manejar los temas sociales de la enfermedad, la normalidad, la mortalidad, los estilos de vida y la filosofia de la vida acorde a nutestro conocimiento de cómo funciona el cerebro y con ello poder ayudar a definir mejor propiamente lo que significa ser bumano y cómo podemos $y$ debemos interacionar socialmente. Es un esfuerzo de cómo alcanzar una filosofía de vida basada en el cerebro...".

Por su parte, la neurociencia de la ética permite el estudio de los circuitos cerebrales y su actividad que dan como consecuencia al ser ético y moral. Su esencia está en que depende del funcionamiento cerebral y más especificamente de ciertos sistemas cerebrales laborando en un contexto social. La conducta moral es la elaboración mental, fruto de la participación de múltiples sistemas neuronales ampliamente distribuidos en el cerebro y que unas veces producen conductas "morales" y, en otras oportunidades distintos tipos de conductas. Participan distintas áreas del cerebro, como el sistema límbico (memoria y sentimientos), el hipocampo y la corteza cerebral (memoria), diversas áreas de asociación de la corteza cerebral (toma de decisiones, responsabilidad, cognición moral). Sin embargo, hay una estructura cerebral clave, que participa en la conducta ética, y es el lóbulo frontal. Más aún, algunos componentes del mismo cumplen funciones más especificas, como la parte más anterior del polo prefrontal, la parte más medial y lateral de la corteza orbitofrontal, la corteza frontal dorsolateral (principalmente en el hemisferio derecho y las partes ventromediales de esa corteza prefrontal.

\section{Cerebro estético}

A la actividad artística de los seres humanos se le debe considerar dentro de una perspectiva neurohistórica, pues su desarrollo sigue un rumbo evolutivo. La obra de arte es un producto del cerebro que busca una comunicación intersubjetiva entre los seres humanos, que implica motivaciones y emociones, en armonía con la razón. El arte es importante para el refuerzo del vínculo social, debido a la universalidad de los modos de comunicación intersubjetiva que implica. Surge, de esto, el interrogarse sobre las bases neurales de la contemplación de la obra de arte, así como también sobre las bases neurales de quien la produce.

Para Jean-Pierre Changeux", "...las funciones cognitivas, en particular la conciencia y la actividad artistica, están asociadas a un desarrollo mayor de la organización cerebral, que se manifiesta, principalmente, por la expansión de la corteza cerebral y, más particularmente, de las cortezas de asociación prefrontal, parietotemporal y cingular, en estrecha relación con el sistema limbico..."

\section{Cerebro religioso/místico}

Dice Carrel "...el sentido moral, la belleza y la mistica únicamente se aprenden cuando se ballan presentes en nuestro ambiente y forman parte de nuestra vida diaria...". Sin embargo, que poco se conoce de la experiencia mística. La tendencia al misticismo es rara. La humanidad tiene una profunda inspiración religiosa, y a pesar de ello, la "actividad mistica ha sido desterrada de la major parte de religiones...". "Requiere una elevaciön espiritual bacia un ser que es manantial de todas las cosas, hacia un poder; un centro de fuerzas, que el mistico llama Dios..."

El éxtasis mistico, como experiencia humana que es, debe tener una base cerebral. El modelo explicativo más conocido de la experiencia mística es el modelo del lóbulo temporal. Este lóbulo reúne más sintomas de tipo afectivo, emocional cuando se lesiona o activa. Rubia ${ }^{10}$ menciona que "....Muchos de los sintomas característicos de la estimulación del lóbulo temporal aparecen también en las experiencias misticas, como la impresión de estar fuera del cuerpo, sensaciones vestibulares (viajar a través del tiempo y del espacio), sensaciones auditivas como escuchar voces de Dios o de seres espirituales que dan consejos, alteraciones perceptivas como 
Iuces brillantes, y también sensaciones de paz, tranquilidad, alegria y bienaventuranza..."

\subsection{El cerebro social}

De manera general, se puede decir que la corteza prefrontal (CPF), así como sus vías hacia las estructuras emocionales del cerebro, es la que produce la inteligencia en la conducta social humana.

La cognición social es la capacidad cerebral a través de las funciones mentales implicadas en la comprensión del mundo social. La revolución cognitiva, entre los años $1960 \mathrm{y}$ 1970, planteaba la metáfora del procesamiento de la información, que plantea la existencia de una serie de procesos mentales por los que fluiría la información dando lugar a la cognición, entre ellas la cognición social. Se puso énfasis en las representaciones mentales. Esto resultaba muy radical por lo que más luego, la cognición social no sólo incluía estas representaciones mentales sino también otros procesos cognitivos generales y específicos. Como resultado del procesamiento de la información obtenemos juicios, comportamientos, reacciones afectivas y decisiones. Algo importante de destacar, es que muchos de estos eventos se llevan a cabo de manera automática, lo que tiene gran influencia en nuestras respuestas, repercutiendo en la adaptación a nuestro entorno social.

En realidad, la cognición social es un proceso sumamente complejo, y una de las formas de procesamiento de información que nos interesa es la atribución. Un concepto interesante, en este ámbito, es la metarrepresentación, es decir, la representación de una representación. Esta adopta dos formas básicas. Cuando se utilizan en agentes distintos de nosotros mismos se llama "lectura de mentes" (mindreading), o también atribución de representaciones en tercera persona. También se pueden realizar atribuciones de representaciones en primera persona, en donde nos vemos como agentes a nosotros mismos, y a esto se denomina metacognición. La metarrepresentación es un formidable instrumento de la sociabilidad humana, y es considerada tan potente como el planteamiento de hipótesis científicas. La "teoria de la mente" (theory of mind) o psicologia folk hacen referencia al conocimiento común, podría decirse intuitivo, que tenemos sobre la relación entre los contenidos de la mente y el comportamiento de los agentes, incluyéndonos a nosotros mismos.

Es importante destacar, que todo el conocimiento que pretendemos tener de las mentes de los demás es solamente una proyección de lo que hemos simulado, en nosotros mismos, como la situación del otro. Solo el conocimiento de nuestras propias mentes sería directo y menos falible, esto es la metacognición. Hasta aquí, lo centrado en los procesos cognitivos implicados en la construcción de representaciones mentales sobre los otros y sobre nosotros mismos, lo cual es fundamental para la cognición social.

También, existen otras teorías que se basan en los procesos sociales que permitirian emerger aquellas capacidades mentales necesarias para la cognición social. Son también llamadas teorias relacionales. Una de ellas es la mentalización que, a su vez, se apoya en la teoria del apego.

La capacidad para la mentalización requiere una serie de habilidades que permiten a la persona interpretar su propia conducta y la de los demás a partir de la atribución de estados mentales tales como sentimientos, creencias, deseos y otros. El apego seguro es importante para que se consolide la mentalización en el niño, y para ello es necesario que los cuidadores sean capaces de leer las señales del bebé y respondan a ellas de manera adecuada. Una labor esencial del cuidador es amplificar las expresiones emocionales positivas del niño y modular las negativas. El niño hace uso de las funciones ya maduras del cerebro adulto para organizar sus propios estados mentales. Y es gracias a esos encuentros interpersonales que logran desarrollar varias funciones mentales esenciales como la memoria, las emociones, la narración y los estados mentales.

\section{El desarrollo filogenético del cerebro social}

Darwin propuso que las facultades sociales humanas tenian una historia evolutiva. Hoy, gracias al desarrollo de la tecnología, especialmente la neuroimagen, es posible explorar las áreas del cerebro relacionadas al ámbito social, tanto a nivel estructural como funcional. El cerebro social humano emerge de un grupo de respuestas emocionales innatas que interactúan con procesos cognitivos de nivel superior. Estas emociones son memorias evolutivas que propician la anticipación y enfrentar con éxito las experiencias que amenazan o favorecen la supervivencia.

Hay un sistema de elevada complejidad que permite atribuir estados mentales a los demás, es el sistema de neuronas espejo, el cual se conecta con el surco temporal superior. Las neuronas espejo son un tipo peculiar de neuronas motoras que codifican actos motores que contienen una meta. Se considera que a partir de estas neuronas es posible descubrir las intenciones de los demás mediante un proceso que permite experimentar los actos del otro como si fueran propios. Las regiones del cerebro parietal y frontal constituyen el núcleo de las neuronas espejo. La peculiaridad de captar la intención de los demás es un aspecto esencial para la interacción social. Es muy probable que también diversas emociones estén mediadas por las neuronas espejo, lo cual sentaría las bases para el desarrollo de las relaciones interpersonales.

Por todo lo dicho, el poder predecir las intenciones de los demás y de esta manera obtener el mayor beneficio posible para uno, tiene como consecuencia una gran utilidad adaptativa. Esta capacidad, llamada inteligencia maquiavélica ${ }^{12}$ ${ }^{13}$, se ha desarrollado en los seres humanos debido a la manera en que usualmente predecimos el comportamiento de los demás, y en ello parecen estar involucradas las neuronas espejo.

\section{REFERENCIAS BIBLIOGRÁFICAS}

1. Carrel Alexis. La incógnita del hombre. 1ra. ed. Editorial Diana 1955.

2. Sagan Caarl. El cerebro de Broca 2da. ed. Drakontos bolsillo 2009 .

3. Kandel Eric. La era del inconciente 1 ra. ed. Paidós. 2013.

Kaku Michio. El futuro de nuestra mente 1ra. ed. Debate 2014.

4. Golberg Elkhonon. El cerebro ejecutivo 2da. ed. Drakontos 2004 . 


\section{Ciencia \& Desarrollo}

Cuya C., El Cerebro Humano, una Perspectiva Transdisciplinaria

5. Peña-Casanova Jordi. Neurología de la conducta y neuropsicología 1ra.ed. Panamericana editorial médica 2007.

7. Churchland Patricia. El cerebro moral 1ra. ed. Paidós 2012.

8. Gazzaniga Michael, El cerebro ético 1ra. ed. Paidós 2006.

9. Changeux Jean-Pierre. Sobre lo verdadero, lo bello y el bien. Un nuevo enfoque neuronal 1 ra. ed. Katz editores
2010.

10. Rubia Francisco. La conexión divina. La experiencia mística y la neurobiología 1ra. ed. Drakontos 2010.

11. Quintanilla Pablo, Mantilla Carla, Cépeda Paola (editores). Cognición social y lenguaje 1 ra. ed. 2014.

12. Gazzaniga Michael. ¿Qué nos hace humanos? 1 ra. ed. Paidós 2010.

13. Brockman John (ed). Mente 1ra. ed. Crítica 2012.

\section{Correspondencia:}

Carlos Cuya Mamani: carlosyovanicuya@yahoo.com.mx
Fecha de Recepción: 03/07/2014

Fecha de Aceptación: 09/07/2014 


\section{NORMAS EDITORIALES Y GUÍA DE PRESENTACIÓN DE ARTICULOS CIENTÍFICOS PARA LA REVISTA CIENCIA \& DESARROLLO}

La Revista Cientifica Ciencia \& Desarrollo pone a consideración de la comunidad cientifica y académica, sus normas editoriales y guia de presentación de los artículos científicos, con la finalidad de lograr que la publicación de la revista sea un espacio abierto para la divulgación de nuevos conocimientos.

\section{CRITERIOS DE PUBLICACIÓN, CITACIÓNY REPRODUCCIÓN}

La revista Ciencia \& Desarrollo es una publicación semestral de carácter científico tecnológico, orientada a promover y difundir la investigación en campos multidisciplinarios. Su publicación es impresa y en idioma español.

Para la publicación en la revista Ciencia \& Desarrollo los artículos presentados deben ser inéditos y pueden ser: artículo científico original, artículo de revisión, carta al editor, reporte de caso clinico, artículo de opinión, reseña de libro, ensayos, artículo breve. Los artículos publicados en esta revista pueden ser citados en otros documentos, siempre y cuando se indique la siguiente información: Revista Científica Ciencia \& Desarrollo. Tacna (Perú): Universidad Nacional Jorge Basadre Grohmann, número de la revista, página(s) y año de publicación. ISSN 2304-8891. Si la reproducción de artículos publicados en la revista, es con fines académicos y sin ánimo de lucro, puede realizarse si se incluye la información establecida en el párrafo anterior. Si su reproducción implica otros usos, debe solicitarse autorización escrita al Director de la Revista cientifica Ciencia \& Desarrollo.

La revista señala que la publicación de artículos no da derecho a remuneración alguna y que la responsabilidad del contenido de los artículos es de los autores, inclusive en lo relacionado con la propiedad intelectual de otros autores $y / o$ fuentes.

La revista cientifica Ciencia \& Desarrollo recibe artículos de las ciencias básicas y aplicadas, tecnológicas y de ingenieria.

\section{TIPOS DEARTÍCULOS}

2.1. Articulo cientifico original: La extensión del desarrollo no debe ser más de 12 páginas o caras. La redacción es en tercera persona. Dado que la finalidad de un artículo científico es comunicar los resultados de investigaciones, ideas y debates de una manera clara, concisa y fidedigna, se tomará en cuenta los principios fundamentales de la redacción científica: precisión, claridad y brevedad. Además de los componentes del articulo cientifico deben cumplir con las siguientes caracteristicas.

Su estructura es del siguiente tipo

Titulo: Debe contener en esencia el problema que está planteado, ser claro y preciso. Consta de 10 a 15 palabras, en caso de ser muy largo utilizar un subtítulo conciso. Su redacción es en mayúscula, respetando la redacción de las denominaciones de nombres científicos.

Title: Es el título traducido correctamente en idioma inglés.

Autor/es: Si el trabajo ha sido realizado en equipo, debe colocarse como primer autor, el que tuvo mayor responsabilidad en la realización del trabajo.

Afiliación. Institución a la cual pertenecen los autores (especificar departamento o área por cada autor)

Resumen: Debe contener en forma escueta los objetivos, planteamiento del problema, materiales y métodos, y resultados. El resumen y abstract se redactan en un promedio de 200 a 250 palabras. Se redacta en tiempo pasado. Debe ser escrito en un solo párrafo, separando las oraciones con punto seguido. Sólo las palabras clave deben ir como punto aparte.

Palabras clave, No deben ser menores a dos ni mayores a cinco, ordenadas alfabéticamente.

Abstract. Es el resumen con una correcta traducción al inglés.

Key Word. Palabras clave correctamente traducidas al inglés.

Introducción: El autor debe procurar que todo el trabajo forme una unidad desde el inicio hasta el final, coherente y rclacionado. En esta parte se expone el problema, se informa lo que se conoce del mismo, se hará una revisión bibliográfica pertinente, se discute el objetivo general, fin principal y la hipótesis (si lo hubiera).

Material y Métodos: Descripción de los recursos humanos, materiales fisicos, financieros y técnico administrativos empleados; explicación detallada del método empleado, señalando las técnicas estadísticas, si se utilizaron.

Resultados: Se expondrá en forma clara siguiendo una secuencia lógica. Se exponen mediante figuras o tablas, donde se exponen la información resumida que confirman o no la(s) hipótesis y objetivos específicos planteados en la investigación. Deben limitarse a describir los propios hallazgos encontrados, evitando adelantar interpretaciones o comparaciones. Se redacta en pasado.

Discusión: En esta parte el autor podrá comparar, discutir, argumentar sus resultados con el de otros autores. Tomar en cuenta que los resultados se exponen y no se recapitulan. De esta discusión se originarán puntos de partida para nuevas investigaciones. Se redacta en presente.

Conclusiones: Deberán ser redactadas en forma clara y concisa.

Agradecimiento: Incluirlos solamente si los hubiera, y solo se menciona a quienes contribuyeron con un apoyo muy importante (técnico) o las instituciones que han financiado la investigación (si fuera el caso).

Referencias Bibliográficas: Deben aparecer solamente las referencias bibliográficas utilizadas por el autor $\mathrm{cn}$ la realización de la investigación y que son mencionadas en la redacción del articulo científico. Serán presentadas en el orden correspondiente al estilo bibliográfico. Considerar que si en el artículo hay quince citas, también debe haber quince referencias bibliográficas.

2.2. Articulo de Revisión: Debe contener: Título en español e inglés; Autor(es); Resumen; Palabras Clave; Abstract; Key Word; Introducción, Método utilizado para localizar y seleccionar los articulos relevantes sobre el tema. Análisis y comparación de los resultados encontrados, coincidencias y discrepancias; conclusiones; recomendaciones; referencias bibliogrăficas.

2.3. Reporte de Caso Clínico: Debe contener: Título en español y en inglés, Autor(es), Resumen, Palabras clave, Abstract, Key Word, Introducción, Anamnesis, Examen clínico, Exámenes auxiliares (laboratorio y gabinete), Etiologia, Diagnóstico preliminar (presuntivo), Tratamiento, Evolución y complicaciones (si las hubiera), Diagnóstico definitivo, Histopatología (si las tuviera), Discusión, Referencias Bibliográficas, Fotografias antes y después del tratamiento. En las fotos del rostro de una persona debe cubrirse los ojos para preservar su identidad, para mostrar el rostro completo se debe contar con el consentimiento escrito, firmado por el paciente y con impresión dactilar de su dedo indice.

2.4. Artículo de Opinión: Debe contener; Título en espanol y en inglés; nombre y apellidos del Autor(es), Introducción, Conclusiones, Referencias Bibliográficas.

2.5. Reseña de Libro: De contenido abierto en cuanto al contenido de la reseña. Sin embargo, será obligatorio considerar: Título del libro en mayúsculas, el nombre del autor o autores (en minúsculas, excepto las primeras letras del nombre y apellido), la edición, la editorial, el año de publicación, el ISBN (si tuviera) y el númcro de páginas. Asi mismo, la carátula del libro, la editorial (escancado a colores). El contenido no debe exceder de dos páginas. Al final de la reseña se debe colocar el nombre del autor de la reseña, indicando además, la licenciatura y los grados académicos (si los tuviera), asi como los principales cargos académicos o institucionales que desempeña.

2.6. Ensayo Científico y Articulo Informativo: La estructura es: Titulo en espanol o inglés; nombre y apellidos del Autor(es); Resumen y Palabras Clave; 
Abstract y Key Words; Introducción; Desarrollo Temático; Conclusiones; Referencias Bibliográficas.

2.7. Carta al Editor: Debe contener: Título en español y en inglés, Autor y filiación institucional, Inicio mencionando la razón objetivo de la carta; si fuera necesario, sólo una tabla o una figura; Razón del planteamiento de la opinión, Discusión de resultados y/o recomendaciones, Referencias Bibliográficas, no más de seis referencias bibliográficas. Correo electrónico.

\section{ESTILO DE PRESENTACIÓN}

Los articulos a ser publicados, deberán cumplir los requisitos que a continuación se detalla:

DE LA PRESENTACIÓN: Se presentará 01 ejemplar impreso de un aproximado de 12 páginas y un CD. Los artículos deber ser preparados en formato MS Word utilizando la fuente Times New Romas de tamaño 10, a espacio sencillo. La primera página del artículo debe incluir; El Título (español e inglés), nombre de autor (es), afiliaciones, abstract y palabras clave. Y utilizarán la misma fuente con distinto tamaño y estilo.

DEL TAMANOY MÁRGENES: En tamaño A4 $(210 \times 297 \mathrm{~cm})$. Los márgenes deben ser configurados de la siguiente manera: superior e izquierdo: 2.5 $\mathrm{cm}$; inferior y derecho: $2 \mathrm{~cm}$. No se permite agregar pie de página ni encabezados. Las referencias y la bibliografía se agregarán al finalizar el texto.

DEL TITULO: El título será escrito en español y en inglés, en tamaño 16 y 14 respectivamente; nombre(s) de autor(es) en tamaño 12 ; la afiliación o institución/área a la que pertenece, dirección y corrco clectrónico (tamaño 12). El título y autor(es) van en negrita y deben estar centrados.

DEL RESUMEN: Resumen (Abstract) del artículo y palabras clave, español y en inglés. Cada artículo debe incluir un resumen de no más de 200 palabras en la primera página, seguido por una lista de palabras clave. El resumen debe ser conciso y las palabras clave deben estar justificadas de ambos lados (izquierdo y derecho).

DEL TEXTO: Estará escrito en dos columnas.

DE LAS REFERENCIAS Y CITAS: Utilizar el estilo de referencias bibliograficas acorde a su investigación. El formato de las citas y referencias bibliográficas que se utilizará será: en las ciencias de la salud (con excepción de Psicologia) la norma Vancouver; y en las ciencias sociales, ciencias humanas, ciencias naturales y Psicologia, la norma APA.

DE LAS SECCIONES: El titulo de una sección debe estar en fuente Times New Roman, tamaño 10 y en negrita, escrito con letras mayúsculas. Debe estar alineado a la izquierda a partir de la introducción, excepto las referencias bibliográficas.

DE LAS SUBDIVISIONES: El título de las subdivisiones debe estar alineado a la izquierda. La fuente a utilizar es Times New Romas tamaño $10 \mathrm{y}$ en negrita y sólo las letras iniciales de cada palabra serán escritas en mayúscula.

DE LAS FIGURAS O TABLAS: Podrá incluirse fotografias, gráficos, tablas o imágenes, etiquetándolos únicamente como figuras o tablas según convenga.

Las figuras serán identificadas con la etiqueta Figura $\mathbf{N}^{\circ}$, numeradas con números arábigos de manera consecutiva, la cual será ubicada al pie de la figura. Las figuras, deberán adjuntarse adicionalmente como archivo de imagen (jpg o png).

Las tablas scrán identificadas con la etiqueta Tabla $\mathbf{N}^{\circ}$, numeradas con números arábigos de manera consecutiva y ubicada en la cabecera de la tabla de forma centrada.

Las figuras y tablas a utilizar deberán ser insertadas en el punto apropiado del texto, debe ser mencionada en el texto al menos una vez y antes de su aparición.

DEL IDIOMA, ESTILO Y CONTENIDO: Los artículos deben presentarse en español. El título y el resumen están inscritos en español e inglés. La ortografia y puntuación deben escribirse con estilo sencillo y directo. Utilice estructuras simples para las oraciones, así como vocabulario común y básico. Defina o explique el vocabulario técnico con sencillez. Explique acrónimos cuando aparezcan en el tẹto por primera vez. La presentación de unidades de medida y valores numéricos se realizará conforme al Sistema Internacional de Unidades, asimismo, debe utilizarse la coma “," para la separación decimal.

IMPORTANTE: Los autores son responsables de asegurarse que su trabajo sea conducido de una manera responsable y ética. La estructura y estilo son tan importantes como el contenido. Antes de escribir su artículo, le recomendamos que lea la amplia literatura disponible para informarse respecto a cómo escribir un buen artículo técnico.

\section{PROCESO DE RECIBO, SELECCIÓN Y EVALUACIÓN}

\subsection{Recepción de los articulos}

Las convocatorias para la recepción de artículos se cierran el 30 de abril y $30 \mathrm{de} \mathrm{sctiembre} \mathrm{de} \mathrm{cada} \mathrm{año.} \mathrm{Los} \mathrm{artículos} \mathrm{se} \mathrm{entregan} \mathrm{en} \mathrm{formato} \mathrm{impreso} \mathrm{en}$ la Oficina General de Investigación de la Universidad Nacional jorge Basadre Grohmann sito en Ciudad Universitaria Av. Miraflores s/n, Tacna, Perú, o son enviados mediante correo electrónico a la cuenta coin@unjbg.edu.pe.

Junto al artículo (construido en un procesador de textos y guardado en formato doc o .docx) se envian las figuras presentadas en él, debidamente identificadas (en formato jpg o png con alta calidad), los datos de los autores debidamente diligenciados.

El formato impreso debe enviarse mediante carta de presentación acompañada del original impreso en papel, más un CD en formato de procesador de textos.

4.2. Proceso de selección

Para que un artículo sea admitido en la revista Ciencia \& Desarrollo debe cumplir con los parámetros de forma establecidos por la revista.

Los autores que decidan retirar sus artículos del proceso de selección, no implica que pierdan la posibilidad de presentarlo para ediciones futuras.

En los articulos que a pesar de ser aceptados, se detecte algún tipo de plagio no serán admitidos para la impresión final.

Después de un tiempo prudencial, el director del comité editorial comunicará al autor sobre la aceptación de su artículo.

4.3. Proceso de Evaluación

La primera evaluación la realiza el Comité Editorial, sobre el cumplimiento de los requisitos formales y la adecuación al interés temático de la Revista, de la cual se envía un mensaje a los autores donde se señala su resultado. En caso de corresponder al interés de la revista, se indican a los autores que aquellos aspectos a ajustar y la fecha límite para hacer la nueva entrega.

Comprobado el cumplimiento de los requisitos formales, cl artículo es enviado a evaluación por parte de árbitros expertos, integrantes del Comité Cientifico de la revista. Este arbitraje se hace de forma confidencial (doble ciego; los árbitros desconocen los nombres de los autores y viceversa), mediante el diligenciamiento de un formato específico, que solicita su concepto sobre la conveniencia o no de su publicación, y las recomendaciones para mejoramiento del artículo. El resultado de la cvaluación se informa a los autores oportunamente mediante correo electrónico, y en caso de requerirse, se indica la fecha para la entrega de los documentos ajustados, con el fin de que los árbitros emitan su concepto definitivo sobre la condición de publicable o no. 